\title{
Impacto socioeconómico por lahares y caída de ceniza ante la erupción del volcán Cayambe en la actividad florícola en Cayambe y Pedro Moncayo
}

\author{
Fernando Pavón* \\ S. Daniel Andrade** \\ Benjamin Bernard** \\ Diana Contreras***
}

Recibido 3 de mayo de 2018; aceptado 8 de octubre de 2018

\begin{abstract}
The complex volcano of Cayambe in Ecuador represents a potential threat, especially for populations that base their economy in floriculture. In this work, we present: 1) spatially modeling the eruption scenarios of the volcano, 2) spatially modeling the potential impact of these risk scenarios by lahar and ash affectation; and 3) evaluate the socioeconomic impacts of eruption scenarios modeled by ash fall through a statistical analysis of numerical simulations of eruptive scenarios and areas affected by lahars taken from the new hazard map of Cayambe. Thus, it was determined that an ash depth of 20 to $50 \mathrm{~mm}$ would be the main issue, covering $81.46 \%$ of the surface with losses amounting to USD 320 million in plants and greenhouses. In the case of lahars, the losses would occur on the grounds. In the case of primary lahars, there might be an estimated loss of USD 45 million and in secondary lahars; the loss might be about USD 15 million. Results obtained in the simulations have a better level of detail than those obtained in previous years by the available cartographic information of the sector and the computational tools utilized. This ties in with Cayambe's depos-

* Escuela Politécnica Nacional, Ladrón de Guevara E11-253 y Andalucía, Quito, Ecuador, correo electrónico: fernando.pavon@epn.edu.ec

** Instituto Geofísico, Escuela Politécnica Nacional, Quito, Ecuador, correos electrónicos: dandrade@igepn.edu.ec; bbernard@igepn.edu.ec

*** Centro de Investigación para la Gestión Integrada del Riesgo de Desastres (CIGIDEN), Av. Vicuña Mackenna 4860, Macul, Santiago, Chile, correo electrónico:

diana.contreras@cigiden.cl
\end{abstract}


its and can be managed for the decision-making in the interest of the affected populations, considering that an eruption of the Cayambe volcano requires careful attention for its high local and national socioeconomic impact because flower exportations are an important economic sector for the country.

Key words: Cayambe, ash, lahar, SIG, socio-economic impact.

\section{Resumen}

El complejo volcánico Cayambe representa una potencial amenaza para poblaciones que basan su economía en la floricultura. En este trabajo, se presenta: 1) modelar espacialmente escenarios de erupción del volcán, 2) modelar espacialmente el potencial impacto de estos escenarios de riesgos por afectación de lahares y de ceniza; y 3) evaluar los impactos socioeconómicos de escenarios de erupción modelados por caída de ceniza mediante un análisis estadístico de simulaciones numéricas de escenarios eruptivos y zonas de afectación por lahares tomadas del nuevo mapa de amenazas del Cayambe. Así se determinó que espesores de ceniza de 20 a 50 mm serían el principal problema cubriendo el $81.46 \%$ de la superficie con pérdidas que ascienden los 300 millones de dólares en plantas e invernaderos. En el caso de lahares las pérdidas se darían en los suelos y se estiman pérdidas de 45 millones de dólares en el caso de lahares primarios y 15 millones de dólares en caso de lahares secundarios. Los resultados obtenidos en las simulaciones tienen mejor nivel de detalle que los obtenidos en años anteriores por la información cartográfica disponible del sector y las herramientas computacionales utilizadas; los mismos que concuerdan con los depósitos del Cayambe y pueden ser manejados para la toma de decisiones en beneficio de las poblaciones afectadas, considerando que una erupción del volcán Cayambe merece una atención especial por su alto impacto socioeconómico local y nacional por ser las exportaciones de flores un rubro importante para el país.

Palabras clave: Lahar, ceniza, SIG, Cayambe, impacto socioeconómico.

\section{Resumo}

O complexo vulcânico Cayambe representa uma potencial ameaça para populações que baseiam sua economia na floricultura. Neste trabalho, se apresenta: 1) modelar espacialmente os cenários de erupção do vulcão; 2) modelar espacialmente o potencial impacto destes cenários de riscos afetados por lahares e cinzas e, 3) avaliar os impactos socioeconômicos de cenários de erupção modelados por queda de cinzas mediante uma análise estadística de simulações numéricas de cenários eruptivos e zonas afetadas por lahares, tomadas pelo novo mapa de ameaças do Cayambe. Assim se determinou que espessuras de cinza de 20 a $50 \mathrm{~mm}$ seriam o principal problema cobrindo $81.46 \%$ da superfície com perdas que ascendem os 300 milhões de dólares (USD) em plantas e estufas. No caso de lahares as perdas se dariam nos solos e se estimam perdas de 45 milhões de dólares no caso de lahares primários e 15 milhões 
no caso de lahares secundários. Os resultados obtidos nas simulações têm melhor nível de detalhe que os obtidos em anos anteriores pela informação cartográfica disponível e as ferramentas computacionais utilizadas; os mesmos que concordam com os depósitos do Cayambe e podem ser manejados para a tomada de decisões em benefício das populações afetadas, considerando que uma erupção do vulcão Cayambe merece uma atenção especial por seu alto impacto socioeconômico local e nacional por ser as exportações de flores um item importante para o país.

Palavras chave: Lahar, cinza, SIG, Cayambe, impacto socioeconômico.

\section{Introducción}

La profundización de las relaciones capitalistas de producción en el sector agrario en Ecuador ha significado, para provincias como la de Pichincha, el crecimiento de actividades agroproductivas de exportación (Martínez, 1994), entre ellas la producción florícola. De manera particular los cantones Cayambe y Pedro Moncayo son los de mayor desarrollo de esta actividad en la provincia de Pichincha. Sin embargo, en estos cantones, las zonas de producción florícola se ubican en los alrededores del complejo volcánico Cayambe, un edificio volcánico que ha presentado significativa actividad en los últimos 11800 años (Hall y Mothes, 1994; Samaniego et al., 1998).

El Cayambe (latitud $00^{\circ} 1.72$ ' norte; longitud $77^{\circ} 59.13^{\prime}$ oeste; altura 5790 metros) se ubica en la parte norte de la Cordillera Real (Oriental) de los Andes del Ecuador, a $60 \mathrm{~km}$ al nororiente de la capital (Quito) y a $15 \mathrm{~km}$ al oriente de la ciudad de Cayambe. El registro de la actividad más reciente del Cayambe incluye de 18 a 20 erupciones en los últimos 4000 años, las mismas que están distribuidas en tres periodos de actividad volcánica (Samaniego et al., 1998): El primer periodo se habría dado entre los años 3800 y 3500 a.p.; el segundo entre 2500 y 1700 años a.p.; y el último inició hace 1100 años a.p. La última erupción del Cayambe ocurrió entre los años 1785 y 1786; se la describe como una erupción subglaciar que habría producido caídas moderadas de ceniza en Cayambe (Samaniego et al., 1998) y la misma habría terminado con un flujo de lava o un lahar en 1786 (Ascázubi, 1802).

Los depósitos observados en los estudios de campo corresponden principalmente a flujos de lava, flujos piroclásticos, lahares y caídas de lapilli y ceniza (Samaniego et al., 2004b). Todos ellos son fenómenos que pueden afectar de forma importante al normal desenvolvimiento de la actividad florícola y, consecuentemente, a la disponibilidad de empleos y a la estabilidad económica del sector. Los vestigios de las erupciones volcánicas en el pasado evidencian el riesgo que una erupción, en la actualidad representaría para las zonas de producción florícola en esta zona, tomando en consi- 
deración que desde el año 2016 ha incrementado la actividad sísmica volcano tectónica registrada en los informes especiales 1, 2, 3, 4 y 5 del 2016 y 1 y 2 del 2017 del Instituto Geofísico de la Escuela Politécnica Nacional (IGEPN, 2016; 2017); así como también la probabilidad de ocurrencia que actualmente posee el volcán Cayambe para el año 2017 es del 68\% (IGEPN, 2017b).

Según Bernard y Samaniego (2017), en caso de reactivación del Cayambe, el escenario eruptivo pesimista más probable corresponde a una erupción explosiva, o a la formación de un domo o un flujo de lava viscosa, en los flancos norte, oriental y occidental. Como consecuencia, lahares primarios se dirigirían al oriente por el sistema fluvial de los ríos Salado-Quijos (Samaniego et al., 2004a) y podrían (dependiendo del tamaño de la erupción) afectar la principal vía de acceso al sector nororiental del país, el Sistema de Oleoductos Trans-Ecuatoriano (SOTE), el Oleoducto de Crudos Pesados (OCP) y al Proyecto Hidroeléctrico Coca-Codo Sinclair.

En cuanto a la caída de ceniza, en esa zona los vientos principalmente van de oriente a occidente, por lo que los asentamientos humanos de la parte occidental podrían verse afectados por este fenómeno (Samaniego et al., 2004b). Este escenario también contempla la posibilidad de que la explosión o crecimiento del domo se produzca en la cumbre o en el flanco occidental, lo que pondría en serio peligro por lahares primarios a la ciudad de Cayambe (Samaniego et al., 2004b) y por caída de ceniza a la actividad florícola.

El propósito de este estudio es delimitar las zonas dedicadas a la actividad florícola que sufrirían una mayor afectación por lahares y caída de ceniza ante una eventual erupción del volcán Cayambe en los cantones Cayambe y Pedro Moncayo (Figura 1) y determinar/cuantificar el impacto socioeconómico en estas zonas a través del análisis de datos censales.

\section{Antecedentes eruptivos y definición de escenarios}

A través de la revisión de estudios previos (Samaniego, 1996; Samaniego et al., 1998; Samaniego et al., 2002; Samaniego et al., 2004a; Samaniego et al., 2004b; Bernard y Samaniego, 2017) se pudo conocer la historia eruptiva del volcán, las fechas de ocurrencia de las principales erupciones, su dinámica y con ello definir escenarios del comportamiento del volcán en eventos futuros, particularmente de los fenómenos de lahares y caída de ceniza. Sin embargo, debido a la falta de información volcanofísica detallada sobre las erupciones recientes del complejo volcánico del Cayambe, para ciertos parámetros como duración, altura de la columna eruptiva, tasa de descarga y volumen de caída se utilizaron los valores definidos por Mastin et al. (2009) con modificaciones para ajustar los resultados a los obtenidos en las turberas en los estudios de Samaniego et al. (1998). 


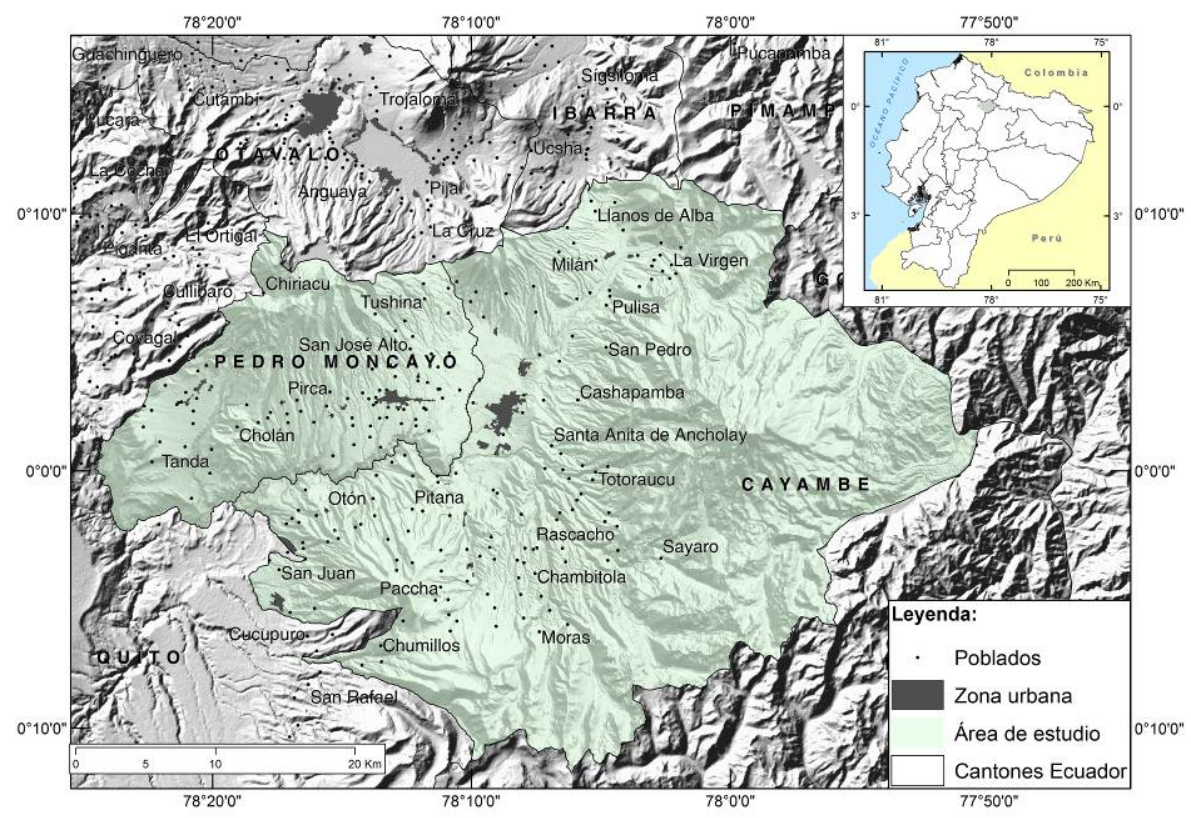

Figura 1. Localización del área en estudio.

Fuente: Modelo de sombras generado a partir de cartografía, IGM, 2012.

Para la determinación de las amenazas asociadas a la caída de ceniza y lahares del volcán Cayambe se representó una erupción volcánica con un índice de explosión volcánica -IEV= 3-4 (tamaño moderado), la cual es caracterizada por la formación de un domo o un flujo de lava viscosa en los flancos norte y oriental (Samaniego et al., 2004b). La creación de un domo podría generar un colapso gravitacional o destrucción explosiva del mismo y con esto, formar nubes ardientes y lahares primarios producto de la fusión parcial del casquete glacial que posee el volcán Cayambe. Este escenario corresponde a una erupción de tipo peleana datada hace 380 años a.p. y se lo conoce como San Marcos-S2 (Samaniego et al., 1998).

Es importante mencionar que la caída de ceniza en zonas pobladas está directamente relacionada con la velocidad y dirección del viento. Los depósitos de nubes ardientes de tipo "bloques y ceniza" cubren una superficie aproximada de $6.9 \mathrm{~km}^{2}$ y tienen un volumen aproximado de $0.4 \mathrm{~km}^{3}$ (Samaniego, 1996). A $4 \mathrm{~km}$ al suroeste de la cumbre del volcán se han identificado depósitos de caída de ceniza que superan los $150 \mathrm{~mm}$. Este tipo de evento llamado San Marcos ha ocurrido en 12 ocasiones con erupciones de tamaño similar en los últimos 4500 años, las cuales pertenecen a ocho periodos eruptivos (Samaniego et al., 1998).

Como ejemplo de erupción de tamaño similar eruptivos ocurridos recientemente en el Ecuador se tomó como umbral bajo a la erupción ocurrida en agosto de 2006 
con el volcán Tungurahua y en umbral alto con la erupción ocurrida en el volcán Reventador en noviembre de 2002 (Bernard y Samaniego, 2017). El escenario S2 corresponde al peor escenario probable; puesto que, el escenario La Chimba-S3 (Bernard y Samaniego, 2017) no se ha presentado en los últimos 10000 años (Samaniego et al., 2004a).

En este sentido, los parámetros utilizados corresponden a un dinamismo eruptivo tipo Peleano o Subpliniano con un $\mathrm{IEV}=3-4$, con duración de seis horas y una altura de la columna eruptiva de $11 \mathrm{~km}$. Con esto lo que se obtiene es la tasa de descarga conocida de $4 \times 10^{6} \mathrm{~kg} / \mathrm{s}$ y un volumen de magma $0.03 \mathrm{~km}^{3}$ (Bernard y Samaniego, 2017).

\section{Materiales y métodos}

\section{Determinación de zonas potencialmente afectadas por lahares}

Para determinar las áreas de afectación de lahares (flujos de lodo y escombros volcánicos), se utilizaron los polígonos derivados en las simulaciones de lahares primarios y secundarios realizados por el IGEPN. Estos resultados fueron obtenidos utilizando modelos digitales de terreno-MDT (resolución de 4 y 10 metros) y el código VolcFlow (Kelfoun y Vallejo, 2015); además se realizaron correcciones topológicas y de escala con el objetivo de obtener un mapa a escala 1:10 000, validados en campo y que son coherentes con fenómenos similares ocurridos en otros volcanes tanto del Ecuador como a nivel mundial (IGEPN, 2017b).

Esta técnica permitió determinar aquellas zonas más susceptibles a ser afectadas por inundaciones de lahares en función de la trayectoria de los ríos de los drenajes occidentales correspondientes a los ríos Guachalá, Purlugara, La Chimba, Blanco y la Quebrada Tunas. Como resultado final de este proceso se obtiene un en formato shapefile, en el cual los polígonos muestran las zonas potencialmente afectadas por lahares primarios y secundarios con sus diferentes volúmenes con la probabilidad de ocurrencia (alta y baja). Para el caso de los lahares primarios, se estima que los volúmenes máximos estarían en el orden de los $15 \times 10^{6} \mathrm{~m}^{3}$ determinado que las zonas que podrían ser potencialmente afectadas corresponden a los drenajes de los ríos Blanco y Guachalá (IGEPN, 2017b). Para el caso de los lahares secundarios, se estima que los volúmenes máximos en los drenajes de los ríos La Chimba y Guachalá serian de $1 \times 10^{6} \mathrm{~m}^{3}$ y para el drenaje del río Blanco $2.5 \times 10^{6} \mathrm{~m}^{3}$ (IGEPN, 2017b).

\section{Determinación de zonas potencialmente afectadas por caída de ceniza}

Para la determinación de zonas potencialmente afectadas por caída de ceniza se utilizó el modelo Ash 3D, el cual simula la dinámica de las nubes de cenizas volcánicas para la zonificación de las áreas potencialmente afectadas por este fenómeno (Mastin et al., 2013). 
Ash 3D es un modelo atmosférico euleriano tridimensional para el transporte, dispersión y deposición de tefra, desarrollado para estudiar y predecir los peligros de las nubes de cenizas volcánicas y la caída de tefra (Mastin et al., 2013). Ash 3D no calcula el comportamiento de una pluma creciente, sino, inyecta tefra en una columna volcánica y los usuarios pueden especificar que las cenizas se concentren en una sola celda, distribuidas uniformemente por toda la columna o distribuidas verticalmente siguiendo la ecuación de Suzuki (1) (Suzuki, 1983; Carey, 1996):

$$
\frac{d Q_{m}}{d y}=Q_{m} \frac{k^{2}\left(1-\frac{Z}{H}\right) \exp \left(k\left(\frac{Z}{H}-1\right)\right)}{H\lfloor 1-(1+k) \exp (-k)\rfloor}
$$

Donde Qm es la masa total del material emitida en un lapso de tiempo con un tamaño de partícula configurado, $H$ es la altura total de la pluma, $z$ corresponde a la elevación en el penacho y el factor de forma $k$, es una constante ajustable que controla la distribución de la ceniza con altura. Valores altos en la constante $k$ concentran la masa cerca de la parte superior del penacho; mientras que, un valor bajo de $k$ da una distribución aproximadamente uniforme de la masa con respecto a la elevación (Mastin et al., 2013).

Para la modelación de caída de ceniza del volcán Cayambe se utilizaron parámetros establecidos anteriormente, con los cuales los resultados obtenidos son concordantes con los descritos en las turberas de los trabajos de campo de Samaniego et al. (1998). Las modelaciones se realizaron para el período 2007-2016, una simulación de erupción el día 15 de cada mes elegido por ser el más representativo, alternando el inicio del evento cada seis horas (00:00, 06:00, 12:00 y 18:00) entre los meses. Con esto se obtuvo un total de 120 modelaciones, con las cuales se realizaron interpolaciones para mejorar las isopacas generadas por el programa. A continuación, se detalla la metodología utilizada para la generación de las isopacas del volcán.

\section{Transformación de los archivos $\mathrm{kmz}$ (resultados de Ash 3D) en shapefile}

Con cada una de las modelaciones generadas en Ash 3D, mediante el uso de los Sistemas de Información Geográfica (SIG), se procedió a la automatización mediante flujos de trabajo, los cuales encadenan secuencias de herramientas de geoprocesamiento, alimentando la salida de una herramienta como entrada en otra herramienta para la transformación de los datos. Esto con la finalidad de replicar el modelo en cada año; puesto que, los archivos originales de salida del código Ash 3D se encuentran en formato kmz. Una vez exportados los archivos a formato shapefile, se eliminó aquella información innecesaria (límite del modelo generado para cada año y mes) para el análisis de los datos. 


\section{Cambio de geometría manteniendo la información de espesores de ceniza}

Eliminados los polígonos correspondientes a los límites de cada modelación, estos fueron transformados a puntos manteniendo los valores de espesores de ceniza de cada uno y posteriormente, se agregó un campo de tipo doble para almacenar la información del espesor del depósito de ceniza porque en primera instancia, se lo obtuvo de tipo texto. El código Ash 3D arroja una serie de datos en cada punto generado; por tal motivo en las modelaciones se tomaron en cuenta los datos finales disponibles en el reporte de cada mes mediante selecciones por atributos, con esto se obtuvieron aquellos datos que cumplan la condición y, posteriormente, se eliminaron los elementos y campos con información no utilizada en el procesamiento.

\section{Automatización del proceso por año}

Con la información depurada, correspondiente a los valores de los puntos extraídos de las modelaciones de Ash 3D mediante el uso de los SIG se generó un modelo para el año 2007 con la finalidad de automatizar el proceso para el periodo 2007-2016, el cual fue ejecutado desde la ventana de Python. Así, lo que se obtuvo es un archivo que servirá como base para interpolar la información generada en cada año del periodo de las modelaciones de caída de ceniza del volcán Cayambe. Posteriormente se agregó un nuevo campo en el cual se almacenó la información de caída de ceniza para los 12 meses y finalmente, se eliminaron aquellos campos innecesarios para el análisis.

\section{Creación de una cuadrícula fija $(200 * 200 \mathrm{~m})$ e interpolación determinista}

Con los límites de las coordenadas de las 120 modelaciones, se generó una cobertura de puntos con una distancia de 200 por 200 metros entre cada uno; esto con la finalidad de mejorar la interpolación de los datos, los cuales posteriormente se utilizaron para obtener las isopacas del volcán Cayambe. Una vez generado el archivo de puntos, se interpoló la información de cada mes para todos los años. Luego, mediante herramientas de geoprocesamiento se obtuvieron los valores de cada año en un par de coordenadas, así en una fila se obtuvo las simulaciones por mes y año por separado.

\section{Análisis geoestadístico de los resultados para obtener mapas de probabilidades}

Generado el archivo de cada año, el cual contiene la información interpolada por meses, se obtuvo mediante el uso de análisis espacial un solo archivo con las 120 simulaciones, las cuales posteriormente fueron analizadas para obtener la probabilidad de ocurrencia al 50\% de caída de ceniza utilizando la mediada de los datos y con esto se generó el archivo final de las zonas potencialmente afectadas por caída de ceniza del volcán. La probabilidad de ocurrencia al $50 \%$ es una de la más utilizadas en la comunidad científica (Bonadonna et al., 2005; Biass et al., 2016). 
El resultado final de este proceso es un archivo en formato shapefile en donde los polígonos representan las zonas potencialmente afectadas por ceniza en sus distintos espesores (> $80 \mathrm{~mm}, 50 \mathrm{~mm}, 30 \mathrm{~mm}, 20 \mathrm{~mm}, 10 \mathrm{~mm}, 5 \mathrm{~mm}, 3 \mathrm{~mm}, 1 \mathrm{~mm},>0 \mathrm{~mm}$ ).

\section{Mapeo de zonas vulnerables a lahares y caída de ceniza}

Con las zonas potencialmente afectadas tanto por lahares y caída de ceniza, se generó el mapa de zonas vulnerables de ambos fenómenos para los cantones de Cayambe y Pedro Moncayo mediante el uso de análisis espacial y algebra de mapas.

Autores como Yokoyama et al. (1984); Stieltjes y Mirgon (1998), consideran que la población representa el elemento más vulnerable frente a una amenaza, razón por la cual este factor junto con la infraestructura y la actividad económica, han sido considerados para el presente estudio. Con base en los factores establecidos (Tabla 1) para la definición de la vulnerabilidad a las amenazas volcánicas (caída de ceniza y lahares) en los cantones Cayambe y Pedro Moncayo fueron: 1) demografía 2) infraestructura, 3) actividad económica.

Tabla 1

Factores de impacto

\begin{tabular}{clccc}
\hline \multirow{2}{*}{ Factor } & \multicolumn{1}{c}{ Variable } & Fuente & Año & $\begin{array}{c}\text { Escalal } \\
\text { Detalle }\end{array}$ \\
\hline \multirow{2}{*}{ Demografía } & Población Total & INEC $^{1}$ & 2010 & Sector Censal \\
\cline { 2 - 5 } & $\begin{array}{l}\text { Población por área } \\
\text { (urbana y rural) }\end{array}$ & INEC & 2010 & Sector Censal \\
\hline \multirow{2}{*}{ Infraestructura } & $\begin{array}{l}\text { Construcciones dedicadas a } \\
\text { la actividad florícola }\end{array}$ & IGM $^{2}$ & 2012 & $1: 5000$ \\
\hline \multirow{2}{*}{$\begin{array}{c}\text { Actividad } \\
\text { económica }\end{array}$} & Uso y cobertura de la tierra & MAGAP & 2014 & $1: 25000$ \\
\cline { 2 - 5 } & Censo Nacional Florícola & MAGAP & 2010 & $1: 5000$ \\
\hline
\end{tabular}

\section{Identificación de infraestructuras dedicadas a la actividad florícola afectadas por lahares y la caída de ceniza}

La cuantificación de áreas dedicadas a la actividad florícola cuya infraestructura puede verse afectada por lahares y caída de ceniza se determinó mediante la digitalización de edificaciones relacionadas con esta actividad. Se utilizó como insumo la fotografía aérea disponible del sector (IGM, 2012) y comprobación en campo de las

1 Instituto Nacional de Estadísticas y Censos.

2 Instituto Geográfico Militar.

3 Ministerio de Agricultura, Ganadería, Acuacultura y Pesca. 
edificaciones levantadas por métodos cartográficos. En este sentido, mediante fotoidentificación se hizo el levantamiento de las estructuras correspondiente a edificaciones y posteriormente se catalogó la información tomando en consideración los parámetros establecidos, de acuerdo a la tabla de estructuración disponible y elaborada por el ente rector de la información Geográfica-Cartográfica del Ecuador (IGM, 2010).

De esta manera, todos los elementos mayores o iguales a una superficie de $6 \mathrm{~m}^{2}$ fueron digitalizados en un archivo tipo polígono; mientras que los de menor superficie fueron representados en un archivo tipo punto a escala 1:5 000. Se digitalizaron 7045 edificaciones de tipo punto y 14380 de tipo polígono, las cuales se sumaron a las levantadas por métodos fotogramétricos por el IGM. Así, mediante el uso de los SIG, se levantaron un total de 26992 puntos y 34582 polígonos; los cuales fueron utilizados como insumo para el análisis de la infraestructura productiva y edificaciones relacionadas a la actividad florícola en los cantones Cayambe y Pedro Moncayo.

\section{Resultados}

\section{Afectación de la población por caída de ceniza y lahares}

De los 118967 habitantes de los cantones Cayambe y Pedro Moncayo, 69880 se encuentran en la zona rural (58.74\%); mientras que 49087 habitantes en la zona urbana (41.26\%) de acuerdo a los datos del INEC, (2010). Un total de 15272 trabajadores se encuentran directamente relacionados a la actividad florícola en los cantones Cayambe y Pedro Moncayo en lo referente a trabajos en los cultivos de flores, post cosecha, cargos administrativos y de tipo guardianía según los datos obtenidos del Censo Nacional Florícola.

De este valor, el $52.97 \%$ corresponden a hombres; mientras que el porcentaje restante $(47.03 \%)$ a mujeres. La mano de obra masculina predomina ligeramente en el cultivo de flores con 848 trabajadores más que mujeres, llegando a un total de 9990 trabajadores. En lo referente a la post cosecha de las flores, la mano de obra de mujeres es mayor con 357 trabajadoras; es decir 2054 mujeres y 1697 hombres.

Tanto en la parte administrativa, como para guardianía se evidencia que la mano de obra para el hombre es más solicitada con un $58.02 \%$ para la primera y un $88.94 \%$ en los cargos de seguridad.

Por la cantidad de habitantes afectados, es necesario implementar medidas de prevención por parte de las autoridades, con la finalidad de salvaguardar la integridad de los habitantes de los 237 poblados disponibles en la cartografía básica oficial a escala 1:5 000 del IGM. De estos poblados, 35 se verían afectados por caída de cenizas con espesores de $10 \mathrm{~mm}$ (13 133 habitantes), 52 con espesores de $20 \mathrm{~mm}$ (23 790 habitantes), 57 con espesores de $30 \mathrm{~mm}$ (57 081 habitantes) y ocho con espesores de 
$50 \mathrm{~mm}$ (888 habitantes) por encontrarse en zonas donde la división censal se encuentra en zonas proximales a la fuente (Figura 2).

Con respecto a los lahares provenientes del volcán Cayambe, se tendrían 49 poblados afectados por lahares secundarios (probabilidad alta de ocurrencia) y de acuerdo a la división censal del INEC llegarían a una población total de 22267 habitantes. Para una probabilidad baja de ocurrencia (lahares primarios) del volcán Cayambe se tendrían 26 poblados con una población total de 21461 habitantes afectados.

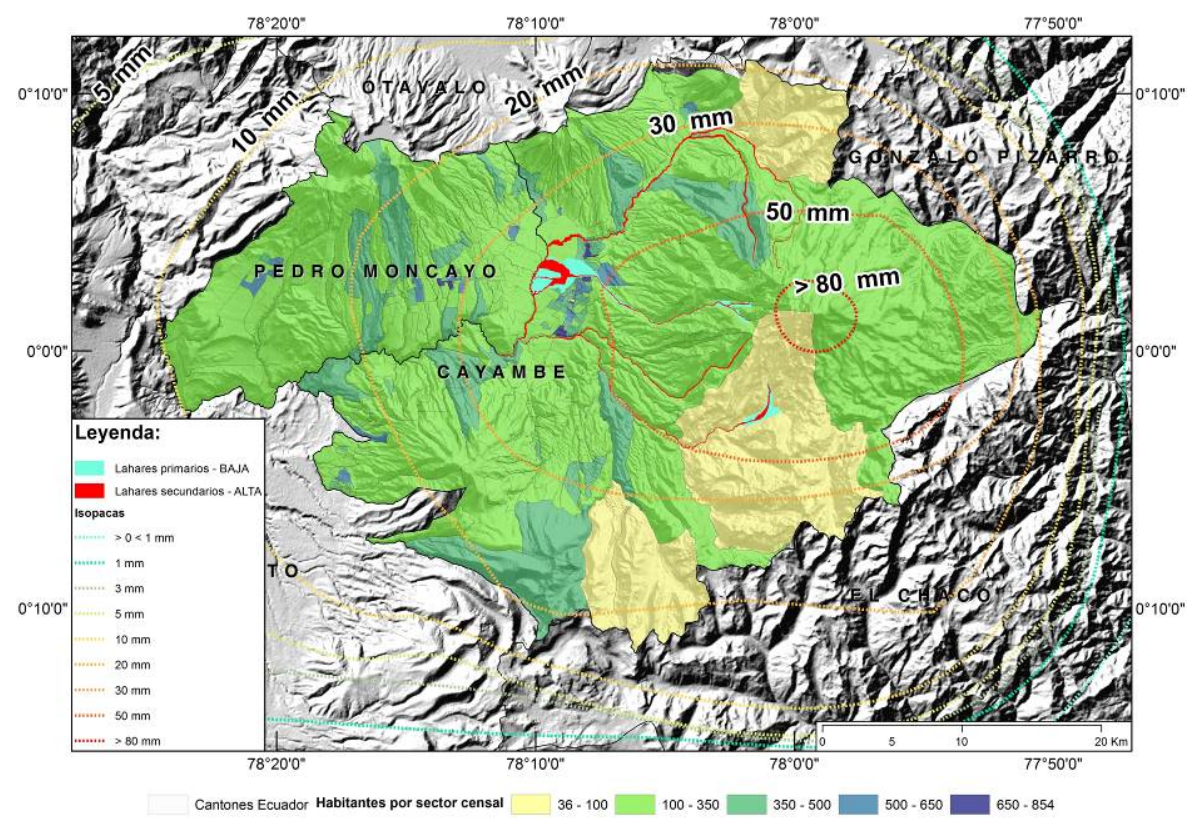

Figura 2. Habitantes afectados por caída de ceniza y lahares.

Fuente: Modelo de sombras generado a partir de cartografía, IGM, 2012.

\section{Afectación por lahares y caída de ceniza según el uso del suelo}

La caracterización del uso de suelo de los cantones Cayambe y Pedro Moncayo, permitió cuantificar la superficie afectada según el uso tanto por lahares como por caída de ceniza. De esta manera, la extensión de los cantones Cayambe y Pedro Moncayo (153 576 ha) en su totalidad se vería afectada por la caída de ceniza proveniente del volcán Cayambe en caso de una erupción de tipo "San Marcos"; siendo los espesores de ceniza de $50 \mathrm{~mm}, 30 \mathrm{~mm}$ y $20 \mathrm{~mm}$ los que cubren el $81.46 \%$ de la superficie de los mismos; mientras que, $1.46 \%$ de la superficie, correspondientes a las áreas proximales al cráter del volcán se verían afectadas con espesores superiores a $80 \mathrm{~mm}$ y el 
$17.05 \%$ de la superficie de los cantones tendrían una afectación con espesores de 5 y $10 \mathrm{~mm}$.

El 52.08\% corresponde a vegetación arbustiva y herbácea con una superficie de 79980 ha, el $32.76 \%$ a tierra agropecuaria con una superficie de 50317 ha. La cobertura de uso de suelo bosque representa el 9.32\% con una superficie de 14320 ha; mientras que, con porcentajes inferiores pero importantes la zona antrópica representa el 3.98\% de la superficie de los cantones (6 $105 \mathrm{ha}$ ), superficies consideradas como otras áreas el 1.60\% (2 455 ha) y cuerpos de agua tan solo el $0.26 \%$ con una superficie de 397 hectáreas (Figura 3).

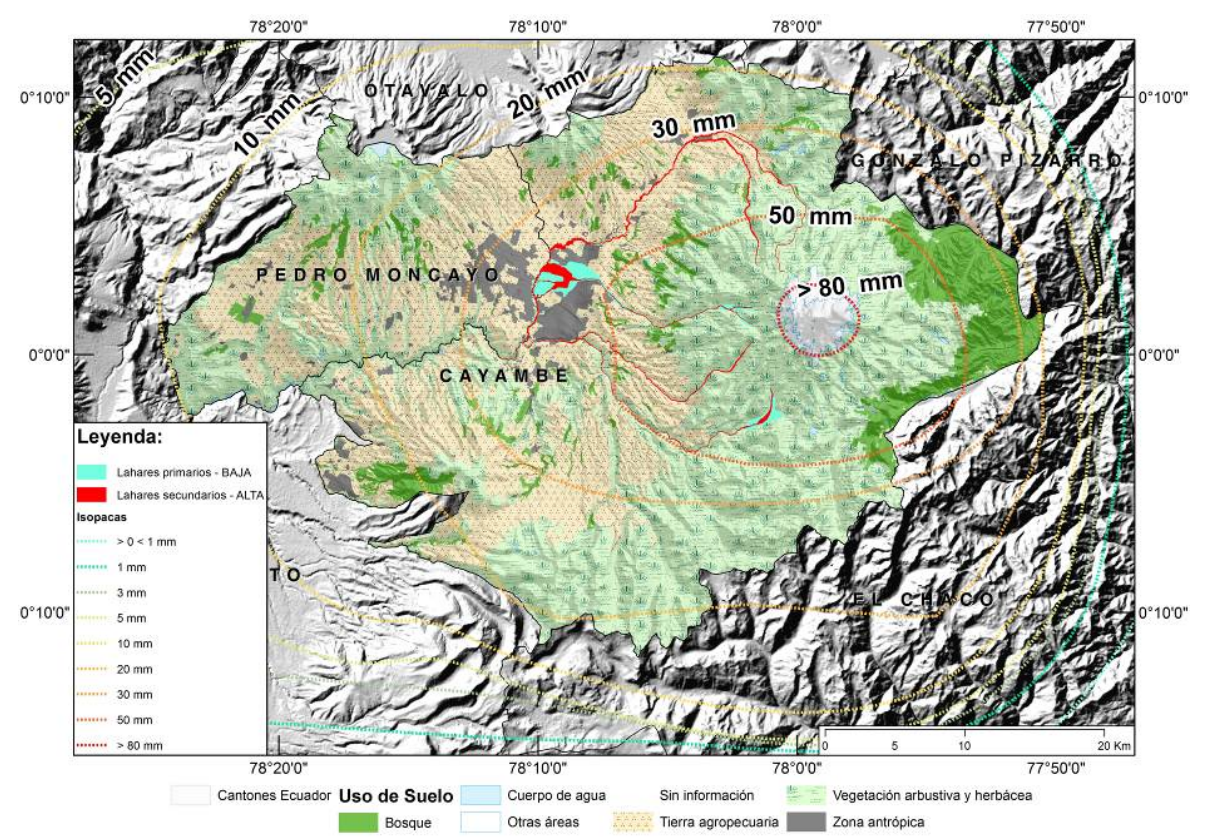

Figura 3. Afectación del uso del suelo por caída de ceniza y lahares.

Fuente: Modelo de sombras generado a partir de cartografía, IGM, 2012.

Un total de 1160 hectáreas de los cantones Cayambe y Pedro Moncayo, correspondientes al $0.76 \%$ de la superficie se verían afectadas por lahares proveniente del volcán Cayambe con una probabilidad de ocurrencia alta (lahares secundarios); mientras que, el $0.98 \%$ tendría una afectación con una probabilidad baja de ocurrencia (lahares primarios), correspondiente a 1511 hectáreas. De la superficie total afectada por lahares, el $44.14 \%$ es vegetación arbustiva y herbácea, $32.57 \%$ está destinado el uso del suelo como zona urbana. Con superficies menores pero importantes, el uso 
de suelo tierra agropecuaria tiene el $25.06 \%$. Coberturas de suelo como bosque representan el $2.06 \%$, y cuerpos de agua $0.16 \%$.

\section{Afectación de infraestructura y edificaciones por caída de ceniza y lahares}

Mediante técnicas cartográficas se pudo levantar un total de 61574 edificaciones en los cantones de Cayambe y Pedro Moncayo; de las cuales mediante foto-identificación se determinaron 13208 edificaciones destinas a la actividad florícola; 4272 han sido catalogados como invernaderos y 8936 como cobertizos. En lo referente a la caída de ceniza, 146 invernaderos y 556 cobertizos se verían afectados con espesores hasta de $50 \mathrm{~mm} ; 2619$ invernaderos y 1574 cobertizos se verían afectados con espesores de $30 \mathrm{~mm}$; 1121 invernaderos y 4391 cobertizos tendrían afectaciones hasta de $20 \mathrm{~mm}$. Por último, 386 invernaderos y 2491 cobertizos estarían afectados con espesores hasta de $10 \mathrm{~mm}$ de caída de ceniza, ante una erupción del volcán Cayambe (Figura 4).

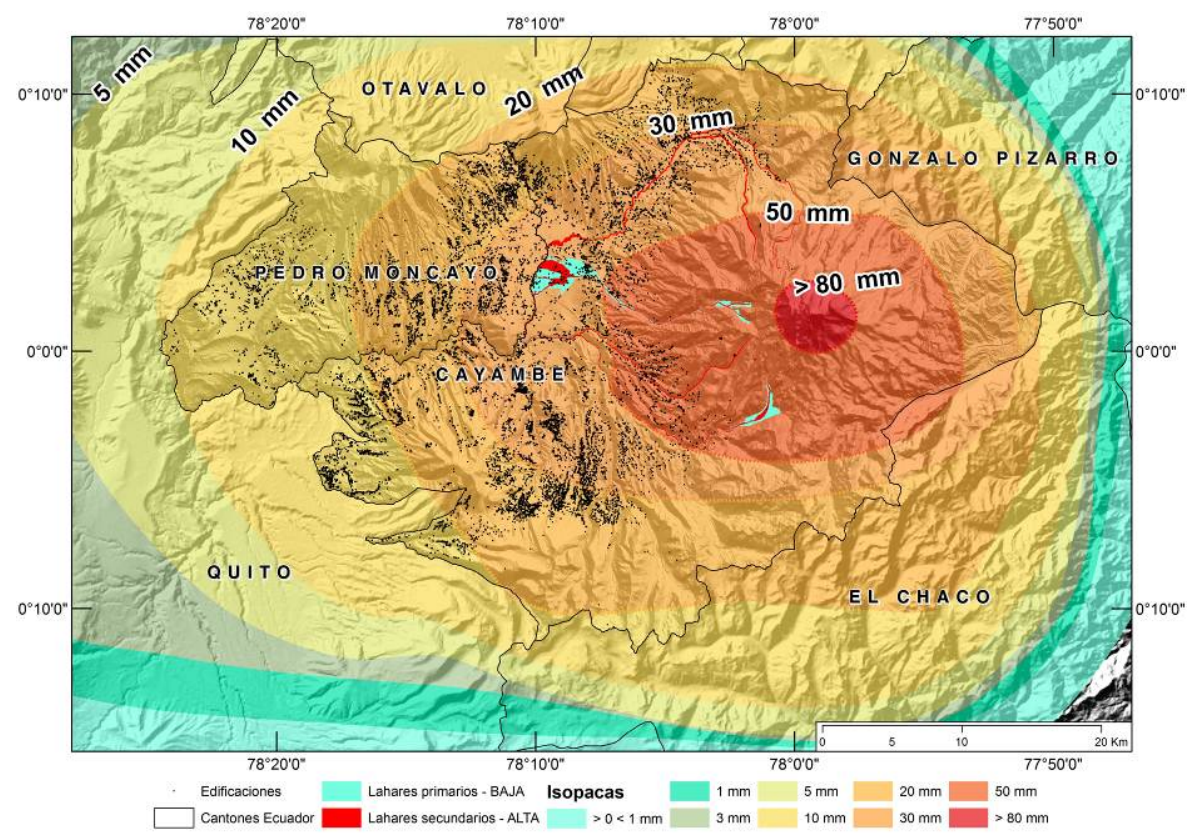

Figura 4. Infraestructura y edificaciones afectadas por caída de ceniza y lahares.

Fuente: Modelo de sombras generado a partir de cartografía, IGM, 2012.

Con respecto a los lahares secundarios (probabilidad alta de ocurrencia) se tendría una afectación de 23 invernaderos, 9 cobertizos y 71 casas; mientras que para lahares primarios (probabilidad de ocurrencia baja), correspondiente a la de mayor extensión 
de lahares se tendría 236 invernaderos, 14 cobertizos y 593 casas directamente afectados. Esto se obtiene tomando en consideración aquellos invernaderos o cobertizos que se encuentran en los límites de las florícolas delimitadas en el Censo Nacional Florícola.

\section{Afectación al sector florícola por caída de ceniza y lahares}

\section{Infraestructura productiva}

La clasificación de las edificaciones permitió enfocar el análisis en aquellas infraestructuras correspondientes a invernaderos y cobertizos, ambas relacionadas con la actividad florícola. Adicionalmente, según el Censo Nacional Florícola del Ministerio de Agricultura y Ganadería (MAGAP, 2009), a escala 1:5 000, en el área de estudio existen unas 152 florícolas con una extensión total de 3217 hectáreas; de las cuales 1434 hectáreas corresponden al cantón Cayambe y 1927 hectáreas al cantón Pedro Moncayo.

Los 152 predios correspondientes a las florícolas de los cantones Cayambe y Pedro Moncayo levantados por el MAGAP en el Censo Nacional Florícola (Figura 5), se los ha dividido en tres grandes grupos según su superficie:

- Pequeños (total de 13): aquellos predios cuya superficie se encuentra entre 0 y menor a 3 hectáreas. 6 florícolas tendrían afectaciones con espesores de $20 \mathrm{~mm}$ y 7 florícolas serían afectadas con espesores de $30 \mathrm{~mm}$.

- Medianos (total de 92): corresponden a superficies de florícolas entre 3 y 20 hectáreas. 8 florícolas tendrían afectaciones con espesores de $10 \mathrm{~mm}, 25$ florícolas serían afectadas con espesores de $20 \mathrm{~mm}$ y 68 florícolas afectadas con espesores $30 \mathrm{~mm}$. Con respecto a la probabilidad de ocurrencia de lahares, 10 florícolas afectadas por lahares primarios; mientras que, 9 serían afectadas por lahares secundarios.

- Grandes (total de 47): predios cuya superficie supera las 20 hectáreas. 3 florícolas se verían afectadas con espesores de $10 \mathrm{~mm}, 7$ florícolas serían afectadas con espesores $20 \mathrm{~mm}, 35$ florícolas podrían verse afectadas con espesores de $30 \mathrm{~mm}$ y 2 florícolas con espesores de $50 \mathrm{~mm}$. Con respecto a la probabilidad de ocurrencia de lahares, 8 florícolas serían afectadas por lahares primarios; mientras que, 9 florícolas serían afectadas por lahares secundarios.

\section{Producción}

Los suelos de los cantones Cayambe y Pedro Moncayo, por sus condiciones favorables, están destinados a la producción florícola, siendo esta actividad uno de los principales ingresos económicos para la zona y también rubro de exportación importante para el país y de acuerdo a los datos disponibles para el año 2014 alcanzó la cifra de 798 millones de dólares (EXPOFLORES, 2015). Según los datos del Censo Nacional 


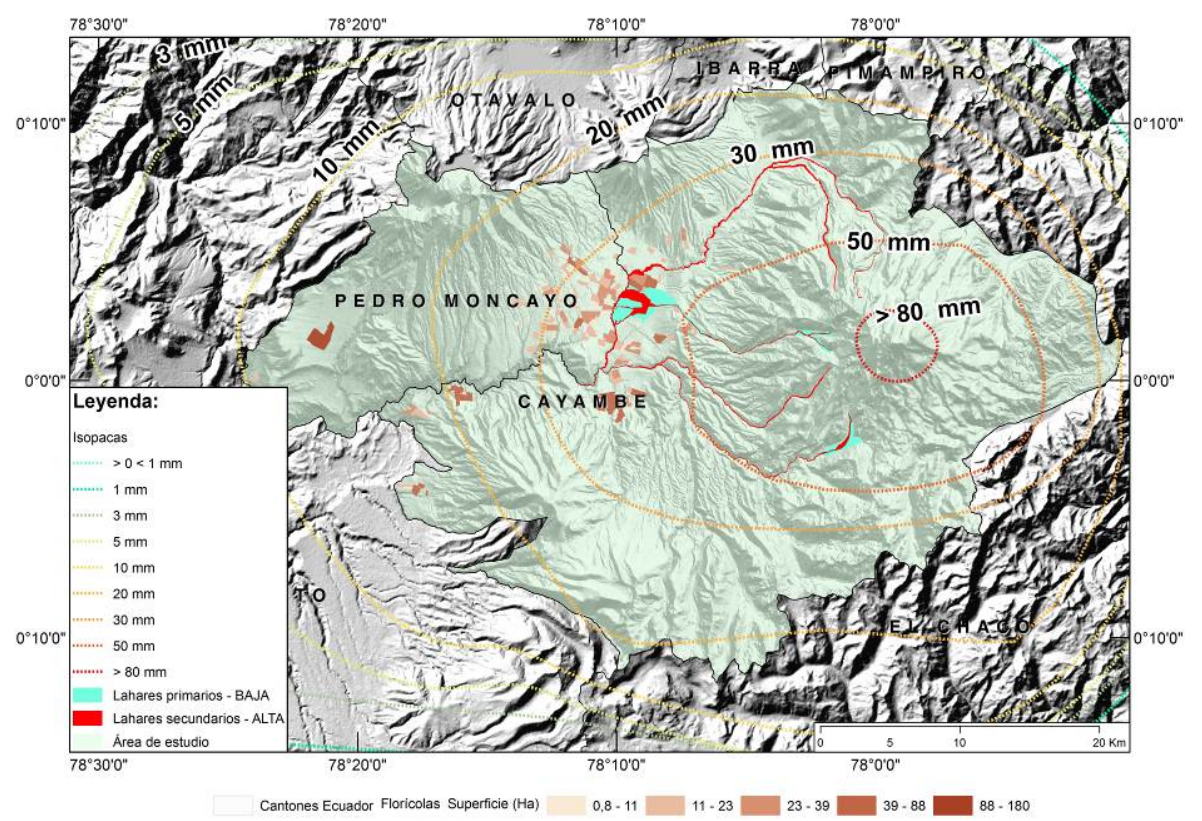

Figura 5. Florícolas potencialmente afectadas por caída de ceniza y lahares.

Fuente: Modelo de sombras generado a partir de cartografía, IGM, 2012.

Florícola (MAGAP, 2009) se evidencia una diversificación de las florícolas según el tipo de flor que se producen en las nueve parroquias dedicadas a esta actividad.

De lo antes expuesto, se demuestra que las florícolas del cantón Cayambe destinan 670 ha para el cultivo de rosas, 25 ha para flor de verano y 24 ha para claveles. Mientras que las florícolas del cantón Pedro Moncayo destinan 707 ha para el cultivo de rosas, 6 ha para flor de verano y 19 ha para claveles.

En el año 2009, el MAGAP determinó el costo aproximado de las florícolas encuestadas en el Censo Nacional Florícola, cuyos valores ascienden a 150000 dólares estadounidenses (USD) por hectárea; de los cuales, el 45\% del valor corresponde a las plantas; seguido por la infraestructura de invernaderos con el $25 \%$, el tercer lugar concierne al valor de la tierra con el $20 \%$ y finalmente, el $10 \%$ representan las construcciones.

Estos valores son considerables en caso de una erupción del volcán Cayambe ya que las florícolas con mayor valor de la provincia de Pichincha se encuentran en los cantones de Cayambe y Pedro Moncayo, potencialmente afectados por caída de ceniza y lahares.

Con los datos obtenidos del Censo Nacional Florícola se determinó que los valores de las florícolas varían entre 27000 hasta 15 millones de dólares, correspondiente 
a las fincas donde se cultivan flores de verano y rosas, respectivamente (MAGAP, 2009). Aproximadamente, el $45 \%$ de las fincas tienen un valor que oscila entre uno y cinco millones de dólares, seguido con el $40 \%$, que les corresponde el grupo de aquellas que tienen un valor entre 1500000 y 1000000 de dólares; mientras que el $12 \%$ tienen valores menores a 150000 ; y finalmente el $3 \%$ tienen valores que superan los 5 millones de dólares (MAGAP, 2009); las cuales tendrían un grado de afectación desde pérdidas parciales a totales en el caso de lahares, dependiendo del evento.

De esta manera, con la información recopilada (MAGAP, 2009) se estimó el costo promedio de pérdidas en las florícolas (Figura 6), en aquellas zonas potencialmente afectadas por ceniza con espesores superiores a $10 \mathrm{~mm}$ las plantas e invernaderos tendrían una afectación considerable (Jenkins et al., 2015), llegando a superar los 200 millones de dólares en pérdidas de plantas y 120 millones de dólares en los invernaderos. En el caso de lahares las pérdidas se dan en los suelos, los cuales serían enterrados por flujos de lodo y con esto, se estiman pérdidas de entre los 15 millones de dólares en el caso de lahares secundarios y 45 millones de dólares adicionales en lahares primarios.

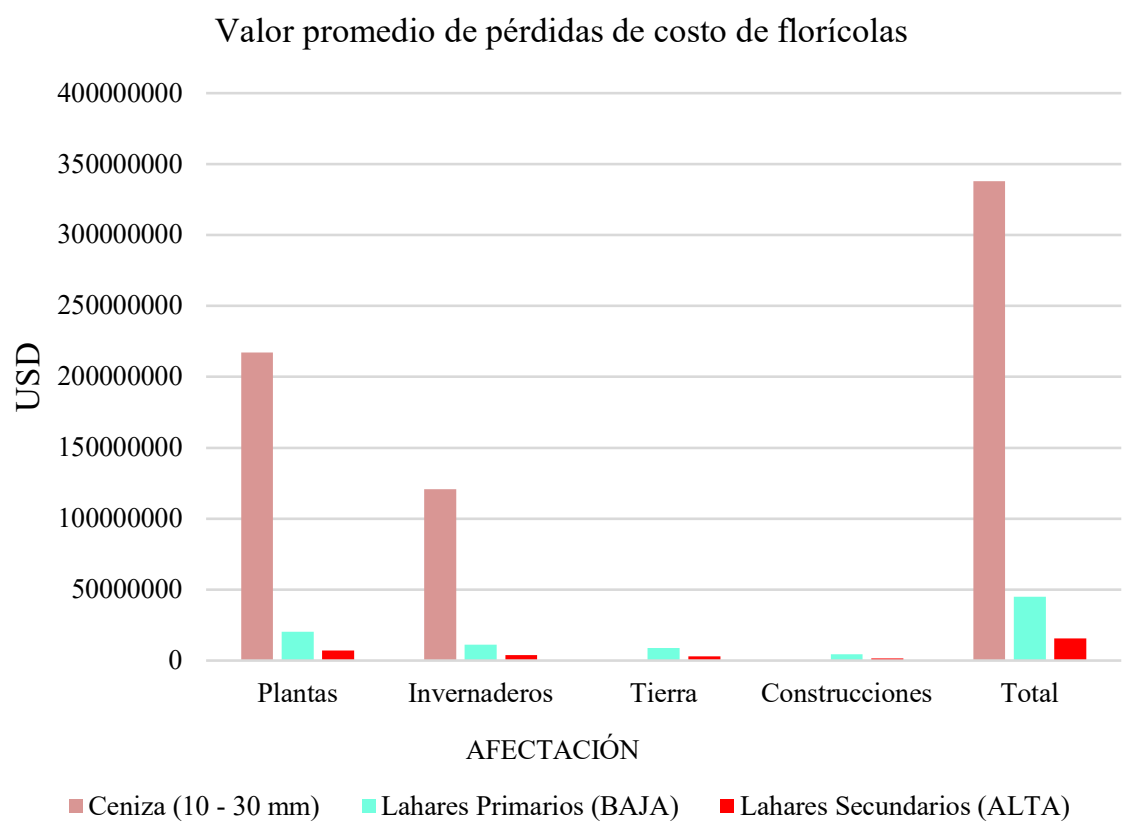

Figura 6. Estimación de pérdidas en costos (USD) por caída de ceniza y lahares. 


\section{Discusión}

Los resultados obtenidos en el presente estudio con respecto a las simulaciones de caída de ceniza mediante el uso del código Ash 3D en el periodo 2007-2016 e interpolaciones de los datos, han permitido la actualización de las amenazas volcánicas relacionadas a cenizas del volcán Cayambe. Dichos datos han sido validados por especialistas del IGEPN para utilizarlos en la actualización del mapa de amenazas del volcán Cayambe; lo que demuestra que el avance de la tecnología e insumos actualizados permiten modelar con mayor precisión los peligros asociados a las amenazas volcánicas y con ello, tener mejores insumos para la toma de decisiones y gestión de riesgos.

Pese a la actualización de la información de la amenaza volcánica, tanto para lahares como caída de ceniza, es necesario mencionar que los datos de carácter socioeconómico disponibles utilizados para este estudio no presentan la misma actualización. Esto ha dificultado una cuantificación más precisa del impacto social y económico en los cantones de Cayambe y Pedro Moncayo ante una eventual erupción. Eso plantea la necesidad de contar con sistemas de información social y económica con una actualización periódica.

Otro aspecto importante es el nivel de detalle de la información cartográfica disponible, la cual solo incluye un levantamiento de las edificaciones, pero no respecto de su estado y año de construcción, aspectos importantes para el análisis de vulnerabilidad de las mismas.

Los datos relacionados con la actividad florícola y sus costos han sido limitados, esto por la falta de apertura de cuentas de los dueños en el Censo Nacional Florícola, de allí que la cuantificación de las pérdidas sea solo referencial y debe ser considerado como valores mínimos. Asimismo, se sugiere que la cuantificación del impacto económico considere la época del año, factor importante si se toma en cuenta que, en ciertas fechas como San Valentín, Día de la madre y Año nuevo, existe un considerable incremento de las ventas para los floricultores.

A pesar de la falta de información social y económica actualizada, el presente estudio sirve para cuantificar el impacto socioeconómico que representa el Cayambe para las poblaciones aleñadas. De igual manera, puede replicarse en otras áreas o diferentes volcanes y con esto, brindar insumos necesarios para la gestión del riesgo y la planificación.

\section{Conclusiones}

El modelo de transporte, dispersión y deposición de tefra, Ash 3D, se utilizó para simular los peligros de las nubes de ceniza y la caída de tefra para el periodo 20072016 y con esto proporcionar una herramienta eficiente y confiable para la evaluación de la amenaza volcánica por caída de ceniza. Después de un análisis cuidadoso del 
modelo, podemos concluir: los tiempos de computación cortos que caracterizan la automatización de procesos también permiten una evaluación totalmente probabilística basada en: 1) un muestreo estocástico de los parámetros de entrada y 2) un análisis probabilístico de los posibles resultados.

Debido a los vientos dominantes con dirección principal hacia el occidente, es probable que sectores ubicados al norte, oriente y sur del Cayambe reciban poca caída de ceniza de una erupción tipo San Marcos. Por lo tanto, ciudades como San Miguel de los Bancos, Sangolquí, Puerto Quito, Pedro Vicente Maldonado, Urcuquí, Pimampiro, El Chaco, Santo Domingo, La Concordia, Lumbaqui y Baeza están relativamente a salvo de la amenaza por caída de tefra del volcán Cayambe.

Las localidades más afectadas son algunas ciudades clave para la producción de flores como Cayambe, Pedro Moncayo y Tabacundo que se encuentran al occidente del volcán Cayambe, que es probable que experimenten daños a la vegetación con espesores de ceniza de $20-50 \mathrm{~mm}$ en el escenario considerado y también el colapso parcial de la infraestructura dedicada a la actividad florícola como los invernaderos y cobertizos de las zonas proximales al volcán.

En lo referente a los lahares, 28947 habitantes afectados, 49 poblados afectados por lahares secundarios con una probabilidad alta de ocurrencia y 26 poblados afectados por lahares primarios con una probabilidad de ocurrencia baja.

El 61\% de los invernaderos (2 619) se verían afectados con espesores de hasta 30 $\mathrm{mm} ; 1121$ construcciones, que representan el $26 \%$ se verían afectadas con espesores de $20 \mathrm{~mm}, 9 \%$ de los invernaderos (386) tendrían afectaciones de hasta $10 \mathrm{~mm}$ y el $3 \%$ (146 invernaderos) estarían afectados con espesores de hasta $50 \mathrm{~mm}$.

Con respecto a los lahares secundarios se tendría una afectación de 103 edificaciones (23 invernaderos, 9 cobertizos y 71 casas); mientras que para lahares primarios se tendría 843 edificaciones directamente afectadas divididas en 236 invernaderos, 14 cobertizos y 593 casas directamente afectados.

Los costos promedio de pérdidas en las florícolas en aquellas zonas potencialmente afectadas por ceniza con espesores superiores a $10 \mathrm{~mm}$, llegando a superar los 200 millones de dólares en pérdidas de plantas y 120 millones de dólares en los invernaderos. En el caso de lahares las pérdidas se dan en las plantas, invernaderos y en los suelos, los cuales serían enterrados por flujos de lodo y con esto, se estiman pérdidas de entre los 15 millones de dólares en el caso de lahares secundarios y 45 millones de dólares en lahares primarios.

La amenaza de dispersión y caída de cenizas volcánicas en el Ecuador es un fenómeno que amerita ser estudiado y monitoreado a profundidad considerando, que en la actualidad las poblaciones dedicadas a actividades primarias como las ganaderas y agrícolas se encuentran ubicadas en zonas proximales a los volcanes por la fertilidad que los suelos presentan por erupciones volcánicas ocurridas en el pasado. 


\section{Agradecimiento}

CONICYT/FONDAP/15110017, Centro de Investigación para la Gestión Integrada de Desastres Naturales (CIGIDEN).

\section{Referencias}

Ascázubi J. (1802). "Letter to Baron von Humboldt", Von Humboldt A, Briefe aus Amerika, 1799-1804, Herausgegeben von Ulrike Moheit, Akademic Verlag, Berlin, 1993.

Bernard B. y Samaniego P. (2017). Escenarios eruptivos en el volcán Cayambe y construcción de un árbol de eventos: Extended abstract volume of the 8th JCT, Quito, Ecuador, p. 5.

Biass S., Bonadonna C., Traglia F., Pistolesi M., Rosi M. and Lestuzzi P. (2016). "Probabilistic evaluation of the physical impact of future tephra fallout events for the Island of Volcano, Italy", Bull Volcanol, 78:37. En 10.1007/s00445-0161028-1.

Bonadonna C., Connor C., Houghton B., Connor L., Byrne M., Laing A. and Hincks T. (2005). "Probabilistic Modeling of Tephra Dispersal: Hazard Assessment of a Multiphase Rhyolitic Eruption at Tarawera, New Zealand", J Geophys Res-Solid Earth. En 110:B03203.

Carey S. (1996). "Modeling of tephra fallout from atmospheric eruptions", in Scarpa, L.A. and Tilling, R.I. (eds.), Monitoring and Mitigation of Volcanic Hazards, Berlin, Springer Verlag, pp. 429-463.

EXPOFLORES -Asociación Nacional de Productores y Exportadores de Flores, (2006).

Hall M. and Mothes P. (1994). Tefroestratigrafía Holocénica de los volcanes principales del Valle Interandino, Ecuador: El contexto geológico del espacio físico Ecuatoriano, R. Marocco (ed.), Corporación Editora Nacional y Colegio de Geógrafos del Ecuador, Quito, Ecuador, pp. 47-67.

IGEPN, Instituto Geofísico de la Escuela Politécnica Nacional (2016). "Informe Especial - Volcán Cayambe $\mathrm{N}^{\mathrm{N}} 1$ ”. Disponible en: $<$ http://www.igepn.edu.ec/cayambe-informes/cay-especiales/cay-e-2016/15310informe-especial-volcan-cayambe-n-1/file>, consultado el 15 de abril de 2017. (2016). "Informe Especial - Volcán Cayambe $\mathrm{N}^{\mathrm{o}} 2$ ". Disponible en: $<$ http://www.igepn.edu.ec/cayambe-informes/cay-especiales/cay-e-2016/16585informe-especial-volcan-cayambe-n-2/file $>$.

(2016). "Informe Especial - Volcán Cayambe $\mathrm{N}^{\mathrm{o}}$ 3". Disponible en: $<$ http://www.igepn.edu.ec/cayambe-informes/cay-especiales/cay-e-2016/16567informe-especial-volcan-cayambe-n-3/file>, consultado el 15 de abril de 2017. 
(2016). "Informe Especial - Volcán Cayambe $\mathrm{N}^{\mathrm{o}}$ 4", disponible en: $<$ http://www.igepn.edu.ec/cayambe-informes/cay-especiales/cay-e-2016/16657informe-especial-volcan-cayambe-n-4/file>, consultado el 15 de abril de 2017.

(2016). "Informe Especial - Volcán Cayambe $\mathrm{N}^{\circ}$ 5", disponible en: $<$ http://www.igepn.edu.ec/cayambe-informes/cay-especiales/cay-e-2016/16780informe-especial-volcan-cayambe-n-5/file>, consultado el 15 de abril de 2017.

(2017). "Informe Especial - Volcán Cayambe $\mathrm{N}^{\circ} 1$ ", disponible en: $<$ http://www.igepn.edu.ec/cayambe-informes/cay-especiales/cay-e-2017/17196informe-especial-volcan-cayambe-n-1-1/file>, consultado el 15 de abril de 2017.

(2017). "Informe Especial - Volcán Cayambe $\mathrm{N}^{\circ} 2$ ", disponible en: $<$ http://www.igepn.edu.ec/cayambe-informes/cay-especiales/cay-e-2017/17425informe-especial-volcan-cayambe-n-1-2/file>, consultado el 15 de abril de 2017. (2017b). Actualización del mapa de amenazas por lahares del volcán Cayambe.

IGM, Instituto Geográfico Militar (2010). Catálogo de objetos del Instituto Geográfico Militar para Cartografia Base Escala 1:5 000, Ecuador.

(2012). Ortofoto Cantones Cayambe y Pedro Moncayo, Ecuador.

INEC, Instituto Nacional de Estadísticas y Censos (2010). VII Censo de Población y VI de Vivienda, Ecuador.

Jenkins S., Wilson T., Magill C., Miller V. and Stewart C. (2015). "Volcanic ash fall hazard and risk: Technical Background Paper for the UNISDR 2015 Global Assessment Report on Disaster Risk Reduction", Global Volcano Model and IAVCEI.

Kelfoun K. and Vallejo S. (2015). VolcFlow capabilities and potencial develpment for the simulation of lava flows.

MAGAP, Ministerio de Agricultura, Ganadería, Acuacultura y Pesca (2009). Censo Nacional Florícola.

Mastin L., Guffanti M., Servranckx R., Webley P., Barsotti S. et al. (2009). "A multidisciplinary effort to assign realistic source parameters to models of volcanic ash-cloud transport and dispersion during eruptions", $J$ Volcanol Geotherm Res, 186:10-21. En: 10.1016/j.jvolgeores.2009.01.008.

Mastin L., Randall M., Schwaiger H. and Denlinger R. (2013). "User's guide and reference to Ash3d-A three-dimensional model for Eulerian atmospheric tephra transport and deposition", U.S. Geological Survey Open-File Report 2013-1122.

Martínez L. (1994). Los campesinos-artesanos en la sierra central: el caso de Tungurahua, CAAP, Quito.

Samaniego P. (1996). "Estudio vulcanológico y petrológico de la historia reciente del volcán Cayambe”, tesis Ing. Geólogo, Escuela Politécnica Nacional. 
Samaniego P., Monzier M., Robin C. and Hall, M.L. (1998). "Late Holocene eruptive activity at Nevado Cayambe Volcano, Ecuador”, Bulletin of Volcanology, 59, pp. 451-459.

Samaniego P., Martin H. and Monzier C. (2002). Transition from calc-alkalic to adakitic magmatism at Cayambe volcano, Ecuador: Insights into slab melts and mantle wedge interactions.

Samaniego P., Robin C., Monzier C., Eissen J. Mothes P. y Hall M. (2004a). El Complejo Volcánico Cayambe, Síntesis geológica, actividad holocénica y evaluación de los peligros volcánicos, Investigaciones en Geociencias, Instituto Geofísico, Escuela Politécnica Nacional, Quito.

Samaniego P., Eissen J., Monzier M., Robin C., Alvarado A. y Yepes H. (2004b). Los peligros volcánicos asociados con el Cayambe, Instituto Geofísico, Quito, 95 pp.

Stieltjes L. et Mirgon C. (1998). “Approche méthodologique de la vulnérabilité aux phénomènes volcaniques. Test d'application sur les réseaux de la Martinique”, Rapport de synthèse. BRGM, R40098, 218 pp.

Suzuki T. (1983). "A theoretical model for dispersion of tephra”, in: D. Shimozuru, I. Yokoyama (eds.), Arc Volcanism: Physics and Tectonics, Terra Scientific Publishing Company (TERRAPUB), Tokyo, pp. 93-113.

Yokoyama I., Tilling R. and Scarpa R. (1984). International mobile early-warning system(s) for volcanic eruptions and related Seismic activities, UNESCO, Paris, FP/2106-82-01(2286), 102 pp. 